\title{
Modified Laplace Decomposition Method for Lane-Emden Type Differential Equations
}

\author{
Fu-Kang Yin, Wang-Yi Han, and Jun-Qiang Song
}

\begin{abstract}
In this paper, A new scheme, deduced from the Modified Laplace decomposition method (MLDM), is presented to obtain exact solutions for the singular initial value problems (IVPs) of lane-Emden type. Both linear and nonlinear cases are considered. If the exact solution exists in the zeroth component, the proposed method yields exact solution in two iterations. The results show that MLDM is very effective and easy to implement.
\end{abstract}

Index Terms-Modified laplace decomposition method, singular, initial value problem, lane-emden type equation.

\section{INTRODUCTION}

Lane-Emden type initial value problems (IVPs) have found a wide range of applications in modeling a class of problems in the field of mathematical physics and astrophysics [1], [2], which can be written in the form:

$$
y^{\prime \prime}+\frac{2}{x} y^{\prime}+f(y)=0, \quad 0 \leq x \leq 1
$$

subject to conditions

$$
y(0)=A, \quad y^{\prime}(0)=B
$$

where $A$ and $B$ are constants. $f(y)$ is a real-valued continuous function. This equation is very useful in the study of various models such as the theory of stellar structure, the thermal behavior of a spherical cloud of gas, isothermal gas spheres and the theory of thermionic currents [1]-[3].

On the other hand, attentions have been paid to another class of singular initial value problems of Lane-Emden type with the form:

$$
y^{\prime \prime}+\frac{2}{x} y^{\prime}+f(x, y)=g(x), \quad 0 \leq x \leq 1
$$

The solution of the Lane-Emden problem is numerically challenging because of the singularity behavior at the origin. The approximate solutions of the Lane-Emden equation were given by Adomian decomposition method [4]-[6], homotopy perturbation method [7]-[9], variational iteration method

Manuscript received September 19, 2012; revised December 24, 2012. This work is supported by National Natural Science Foundation of China (Grant Nos. 41105063).

The authors are with National University of Defense Technology, Changsha, P. R. China (e-mail: yinfukang@nudt.edu.cn, hanwangyige@163.com,junqiang@nudt.edu.cn).
[10]-[12], differential transformation method [13] Wavelets-Collocation method [14], [15] and so on.

Laplace Adomian decomposition method (LADM) was first proposed by Suheil A. Khuri [16], [17] and has been successfully used to find the solution of differential equations [18]-[23]. The major advantage of this method is its capability of combining the two powerful methods to obtain exact solutions for nonlinear equations. However, LADM will generate "noise term" [24] for inhomogeneous equations. Therefore, M. Hussain [25] developed a modified Laplace decomposition method (MLDM) which can accelerate the rapid convergence of series solution when compared with Laplace Adomian decomposition method. In this paper, we will apply the MLDM to obtain exact or approximate analytical solutions of the Lane-Emden type equations.

\section{Modified LAPLACE DeCOMPOSITION METHOD}

In this section, we will briefly discuss the use of the MLDM for the solution of Lane-Emden equation given in (3).

Multiplying $x$ and then taking the Laplace transform on both sides of (3) gives:

$$
-s^{2} L^{\prime}\{y\}-y(0)+L\{x f(x, y)-x g(x)\}=0,
$$

where $L$ is the operator of Laplace transform and $L^{\prime}\{y\}=\frac{d L\{y\}}{d s}$.

We decompose $f(x, y)$ into two parts:

$$
f(x, y)=R[y(x)]+N[y(x)],
$$

where $R[y(x)]$ and $N[y(x)]$ denote the linear term and the nonlinear term respectively.

The Adomian decomposition method and the Adomian polynomials can be used to handle Eq. (4) and to address the nonlinear term $N[y(x)]$. MLDM defines a solution $y(x)$ by an infinite series of components given by:

$$
y(x)=\sum_{n=0}^{\infty} y_{n}(x)
$$

and the nonlinear term can be represented by an infinite series of the Adomian polynomials $A_{n}$ in the form:

$$
N\{y(x)\}=\sum_{n=0}^{\infty} A_{n}(x),
$$


where $A_{n}$ are the Adomian polynomials and it can be calculated by formula given below:

$$
A_{n}=\frac{1}{n !}\left[\frac{d^{n}}{d \lambda^{n}} N\left(\sum_{n=0}^{\infty} \lambda^{n} u_{n}\right)\right]_{\lambda=0}, n=0,1,2, \cdots
$$

Therefore Adomian's polynomials are given by:

$$
\begin{aligned}
& A_{0}=N\left[u_{0}\right], \\
& A_{1}=u_{1} N^{\prime}\left[u_{0}\right], \\
& A_{2}=u_{2} N^{\prime}\left[u_{0}\right]+\frac{1}{2 !} u_{1}^{2} N^{\prime \prime}\left[u_{0}\right], \\
& A_{3}=u_{3} N^{\prime}\left[u_{0}\right]+u_{1} u_{2} N^{\prime \prime}\left[u_{0}\right]+\frac{1}{3 !} u_{1}^{3} N^{\prime \prime \prime}\left[u_{0}\right],
\end{aligned}
$$

After substituting (6) and (7) into (4), we have

$$
\left.\begin{array}{l}
-s^{2} L^{\prime}\left\{\sum_{n=0}^{\infty} y_{n}(x)\right\}-y(0)-L\{x g(x)\} \\
+L\left\{x R\left[\sum_{n=0}^{\infty} y_{n}(x)\right]+x \sum_{n=0}^{\infty} A_{n}(x)\right\}
\end{array}\right\}=0 .
$$

Using the linearity of Laplace transform, it follows that

$$
\left.\begin{array}{l}
-s^{2} \sum_{n=0}^{\infty} L^{\prime}\left\{y_{n}(x)\right\}-y(0)-L\{x g(x)\} \\
+\sum_{n=0}^{\infty} L\left\{x R\left[y_{n}(x)\right]+x A_{n}(x)\right\}
\end{array}\right\}=0,
$$

In general, the recursive relation is given by:

$$
\begin{aligned}
& L^{\prime}\left\{y_{0}(x)\right\}=-s^{-2} y(0)-s^{-2} L\{x g(x)\}, \\
& L^{\prime}\left\{y_{n+1}(x)\right\}=s^{-2} L\left\{x R\left[y_{n}(x)\right]+x A_{n}(x)\right\},
\end{aligned}
$$

By integrating both sides of Eq. (12) from 0 to $s$ respect with s, we have

$$
\begin{aligned}
& L\left\{y_{0}(x)\right\}=\int\left[-s^{-2} y(0)-s^{-2} L\{x g(x)\}\right] d s, \\
& L\left\{y_{n+1}(x)\right\}=\int s^{-2} L\left\{x R\left[y_{n}(x)\right]+x A_{n}(x)\right\} d s,
\end{aligned}
$$

Taking the inverse Laplace transform to Eq. (13), one obtains

$$
\begin{aligned}
& y_{0}(x)=L^{-1}\left\{\int\left[-s^{-2} y(0)-s^{-2} L\{x g(x)\}\right] d s\right\}=H(x), \\
& y_{n+1}(x)=L^{-1}\left\{\int s^{-2} L\left\{x R\left[y_{n}(x)\right]+x A_{n}(x)\right\} d s\right\},
\end{aligned}
$$

where $H(x)$ represents the term arising from source term and prescribed initial condition. The initial solution is important, and the choice of Eq. (14) as the initial solution always leads to noise oscillation during the iteration procedure.

In order overcome the shortcoming, we assume that $H(x)$ can be divided into the sum of two parts namely $H_{0}(x)$ and $H_{1}(x)$, therefore we get

$$
H(x)=H_{0}(x)+H_{1}(x) .
$$

Instead of the iteration procedure Eq. (14), we suggest the following modification

$$
\begin{aligned}
& y_{0}(x)=H_{0}(x) \\
& y_{1}(x)=H_{1}(x)+L^{-1}\left\{\int s^{-2} L\left\{x R\left[y_{n}(x)\right]+x A_{n}(x)\right\} d s\right\}, \\
& y_{n+1}(x)=L^{-1}\left\{\int s^{-2} L\left\{x R\left[y_{n}(x)\right]+x A_{n}(x)\right\} d s\right\}
\end{aligned}
$$

The solution through the modified Laplace decomposition method highly depends upon the choice of $H_{0}(x)$ and $H_{1}(x)$.

\section{ILLUSTRATIVE EXAMPLES}

In this section, we will apply the method presented in this paper to solve singular IVPs of Lane-Emden-type.

\section{A. Linear Cases}

Example 1. Consider the following linear, homogeneous Lane-Emden differential equation $(5,9,11,26,27,29)$

$$
y^{\prime \prime}+\frac{2}{t} y^{\prime}-2\left(2 t^{2}+3\right) y=0
$$

with initial conditions

$$
y(0)=1, \quad y^{\prime}(0)=0 .
$$

According to the MLDM and initial conditions, we get

$$
-s^{2} L^{\prime}\{y\}-1=L\left\{2\left(2 t^{3}+3 t\right) y\right\},
$$

and then, the recursive relations can be obtained as

$$
\begin{aligned}
& y_{0}(t)=L^{-1}\left\{\int\left[-s^{-2} y(0)\right] d s\right\}, \\
& y_{n}(t)=L^{-1}\left\{\int-s^{-2} L\left\{2\left(2 t^{3}+3 t\right) y_{n-1}(t)\right\} d s\right\},
\end{aligned}
$$

Considering equation (20), starting with substituting the value of $y(0)=1$ given in (18) into (20), we have

$$
\begin{aligned}
& y_{0}=1, \\
& y_{1}=t^{2}+\frac{1}{5} t^{4}, \\
& y_{2}=\frac{3}{10} t^{4}+\frac{13}{105} t^{6}+\frac{1}{90} t^{8} \\
& y_{3}=\frac{3}{70} t^{6}+\frac{17}{630} t^{8}+\frac{59}{11550} t^{10}+\frac{1}{3510} t^{12},
\end{aligned}
$$

Hence, the solution series in general gives

$$
y=y_{1}+y_{2}+y_{3}+\cdots=1+t^{2}+\frac{1}{2} t^{4}+\frac{1}{6} t^{6} \cdots
$$

The closed form of the series (22) is $y(t)=\exp \left(t^{2}\right)$ which gives an exact solution of the problem. 
It should be noted that the MLDM gives an analytical solution in the closed form like the Adomian decomposition method [5], homotopy perturbation method [9] and variational iteration method [11].

Example 2. Consider the linear, non-homogenous Lane-Emden differential equation (14)

$$
y^{\prime \prime}+\frac{2}{t} y^{\prime}+y^{n}=0, \quad t \geq 0, \quad n=0,1
$$

subject to initial conditions

$$
y(0)=1, \quad y^{\prime}(0)=0 \text {. }
$$

According to the MLDM and initial conditions (24), we have

$$
-s^{2} L^{\prime}\{y\}-y(0)+L\left\{t y^{n}\right\}=0 .
$$

when $n=0$, we obtain

$$
-s^{2} L^{\prime}\{y\}-y(0)+L\{t\}=0,
$$

We east get

$$
L^{\prime}\{y\}=-\frac{1}{s^{2}}+\frac{1}{s^{4}}, L\{y\}=\frac{1}{s}-\frac{1}{3 s^{3}}, \quad y(t)=1-\frac{t^{2}}{6}
$$

which the exact solution.

When $n=1$, we obtain

$$
-s^{2} L^{\prime}\{y\}-1+L\{t y\}=0,
$$

the recursive relation is obtained as

$$
\begin{aligned}
& y_{0}(t)=L^{-1}\left\{\int\left[-s^{-2} y(0)\right] d s\right\}, \\
& y_{n}(t)=L^{-1}\left\{\int-s^{-2} L\left\{t y_{n-1}(t)\right\} d s\right\},
\end{aligned}
$$

By using Eq. (29), we can get

$$
y_{0}=1, \quad y_{1}=-\frac{t^{2}}{3 \times 2 !}, \quad y_{2}=\frac{t^{4}}{5 \times 4 !}, \quad y_{3}=-\frac{t^{6}}{7 \times 6 !}, \cdots
$$

Hence, the solution series in general gives

$$
y=1-\frac{t^{2}}{3 \times 2 !}+\frac{t^{2}}{5 \times 4 !}-\frac{t^{6}}{7 \times 6 !}+\cdots,
$$

The closed form of the series (31) is $y(t)=\sin t / t$ which gives an exact solution of the problem.

Example 3. Consider the linear, non-homogenous Lane-Emden equation $(5,11,26,27)$ :

$$
y^{\prime \prime}+\frac{2}{t} y^{\prime}+y=6+12 t+t^{2}+t^{3}, \quad 0<t \leq 2
$$

subject to initial conditions

$$
y(0)=0, \quad y^{\prime}(0)=0 .
$$

According to the MLDM and initial conditions (33), we have

$$
-s^{2} L^{\prime}\{y\}+L\{t y\}-L\left\{6 t+12 t^{2}+t^{3}+t^{4}\right\}=0,
$$

the recursive relation is obtained as

$$
\begin{aligned}
& y_{0}(t)=L^{-1}\left\{\int-s^{-2} L\left\{6 t+12 t^{2}\right\} d s\right\}, \\
& y_{1}(t)=L^{-1}\left\{\int s^{-2}\left\{L\left\{t y_{0}\right\}-L\left\{t^{3}+t^{4}\right\}\right\} d s\right\}, \\
& y_{n}(t)=L^{-1}\left\{\int s^{-2} L\left\{t y_{n-1}\right\} d s\right\},
\end{aligned}
$$

And then we have

$$
y_{0}=t^{2}+t^{3}, y_{1}=0, y_{n}=0
$$

so the exact solution is obtained as $y=t^{2}+t^{3}$.

Unlike the variational iteration method [11] which introduces noise terms, our method can get the exact solution while only need a few iterations.

\section{B. Nonlinear Cases}

Example 4. Consider the following nonlinear, homogeneous Lane-Emden differential equation

$$
y^{\prime \prime}+\frac{2}{t} y^{\prime}+y^{3}-\left(6+t^{6}\right)=0, \quad t \geq 0
$$

subject to initial conditions

$$
y(0)=0, \quad y^{\prime}(0)=0 .
$$

According to the MLDM and initial conditions (38), we have

$$
-s^{2} L^{\prime}\{y\}+L\left\{t y^{3}\right\}-L\left\{6 t+t^{7}\right\}=0,
$$

the recursive relation is obtained as

$$
\begin{aligned}
& L^{\prime}\left\{y_{0}\right\}=-s^{-2} L\{6 t\}, \\
& L^{\prime}\left\{y_{1}\right\}=s^{-2} L\left\{t y_{n-1}^{3}\right\}-L\left\{t^{7}\right\}, \\
& L^{\prime}\left\{y_{n}\right\}=s^{-2} L\left\{t y_{n-1}^{3}\right\},
\end{aligned}
$$

so we have

$$
\begin{aligned}
& L\left\{y_{0}\right\}=\frac{2}{s^{3}}, \quad y_{0}=t^{2}, \\
& L\left\{y_{1}\right\}=0, \quad y_{1}=0, \\
& L\left\{y_{n}\right\}=0, \quad y_{n}=0, \quad n>1
\end{aligned}
$$

so the exact solution is obtained as $y=t^{2}$.

Example 5. Consider the following nonlinear, 
homogeneous Lane-Emden differential equation (9, 11, [27]-[29]:

$$
y^{\prime \prime}+\frac{2}{t} y^{\prime}+4\left(2 e^{y}+e^{y / 2}\right)=0, \quad 0 \leq t \leq 1
$$

subject to initial conditions

$$
y(0)=0, \quad y^{\prime}(0)=0
$$

The exact solution is $y(t)=-2 \ln \left(1+t^{2}\right)$.

Applying the MLDM and initial conditions (43), we have

$$
-s^{2} L^{\prime}\{y\}+L\left\{4 t\left(2 e^{y}+e^{y / 2}\right)\right\}=0,
$$

and then, we get the recursive relation as

$$
L^{\prime}\left\{y_{0}\right\}=0, \quad L^{\prime}\left\{y_{n}\right\}=s^{-2} L\left\{4 t A_{n-1}\right\}
$$

where the nonlinear operator $N[y]=2 e^{y}+e^{y / 2}$ is decomposed as in (9) in terms of the Adomian polynomials. From (10) the first few Adomian polynomials for $N[y]=2 e^{y}+e^{y / 2}$ are computed as follows:

$$
\begin{aligned}
A_{0}= & 2 e^{y_{0}}+e^{y_{0} / 2} \\
A_{1}= & y_{1}\left(2 e^{y_{0}}+\frac{1}{2} e^{y_{0} / 2}\right), \\
A_{2}= & y_{2}\left(2 e^{y_{0}}+\frac{1}{2} e^{y_{0} / 2}\right)+\frac{y_{1}^{2}}{2 !}\left(2 e^{y_{0}}+\frac{1}{4} e^{y_{0} / 2}\right), \\
A_{3}= & y_{3}\left(2 e^{y_{0}}+\frac{1}{2} e^{y_{0} / 2}\right)+y_{1} y_{2}\left(2 e^{y_{0}}+\frac{1}{4} e^{y_{0} / 2}\right) \\
& +\frac{y_{1}^{3}}{3 !}\left(2 e^{y_{0}}+\frac{1}{8} e^{y_{0} / 2}\right)
\end{aligned}
$$

And then by using (45), we have

$$
\begin{aligned}
& L^{\prime}\left\{y_{0}\right\}=0, \quad L\left\{y_{0}\right\}=0, \quad L\left\{y_{1}\right\}=-\frac{12 \times 1 !}{3 s^{3}}, \quad y_{1}=-2 t^{2}, \\
& \left.L^{\prime}\left\{y_{1}\right\}=\frac{12 \times 1 !}{s^{4}}, \quad y^{\prime}, \quad y_{2}\right\}=-\frac{20 \times 3 !}{s^{6}}, L\left\{y_{2}\right\}=-\frac{20 \times 3 !}{5 s^{5}}, y_{2}=t^{4}, \\
& L^{\prime}\left\{y_{3}\right\}=\frac{28 \times 5 !}{s^{8}}, L\left\{y_{3}\right\}=-\frac{28 \times 5 !}{7 s^{7}}, y_{3}=-\frac{2}{3} t^{6}, \\
& L^{\prime}\left\{y_{4}\right\}=-\frac{36 \times 7 !}{s^{10}}, L\left\{y_{4}\right\}=\frac{36 \times 7 !}{9 s^{9}}, \quad y_{3}=\frac{1}{2} t^{8},
\end{aligned}
$$

Then the solution in a series form is given by

$$
y(t)=-2\left(t^{2}-\frac{1}{2} t^{4}+\frac{1}{3} t^{6}-\frac{1}{4} t^{8}+\cdots\right) .
$$

Hence the exact solution has the form

$$
y(t)=-2 \ln \left(1+t^{2}\right)
$$

Example 6. Consider the nonlinear, homogeneous Lane-Emden differential equation $(9,11,27)$

$$
y^{\prime \prime}+\frac{2}{t} y^{\prime}-6 y=4 y \ln y, \quad 0 \leq t \leq 1,
$$

subject to initial conditions

$$
y(0)=1, \quad y^{\prime}(0)=0 .
$$

The exact solution is $y(t)=e^{t^{2}}$.

Applying the MLDM and initial conditions (51), we have

$$
-s^{2} L^{\prime}\{y\}-1=L\{6 y t+4 y t \ln y\}
$$

and then, we get the recursive relation as

$$
L^{\prime}\left\{y_{0}\right\}=-s^{-2}, \quad L^{\prime}\left\{y_{n}\right\}=s^{-2} L\left\{6 y_{n-1} t+4 y_{n-1} t \ln y\right\},
$$

where the nonlinear operator $N[y]=y \ln y$ is decomposed as in (9) in terms of the Adomian polynomials. From (10) the first few Adomian polynomials for $N[y]=y \ln y$ are computed as follows:

$$
\begin{aligned}
& A_{0}=y_{0} \ln y_{0}, \\
& A_{1}=y_{1}\left(1+\ln y_{0}\right), \\
& A_{2}=y_{2}\left(1+\ln y_{0}\right)+\frac{y_{1}^{2}}{2 y_{0}}, \\
& A_{3}=y_{3}\left(1+\ln y_{0}\right)+\frac{y_{1} y_{2}}{y_{0}}-\frac{y_{1}^{3}}{6 y_{0}^{2}},
\end{aligned}
$$

According to Eq. (53) and by using the Eq. (54), we can get the series solution as

$$
\begin{aligned}
& L^{\prime}\left\{y_{0}\right\}=-\frac{1}{s^{2}}, \quad L\left\{y_{0}\right\}=\frac{1}{s}, \quad y_{0}=1, \\
& L^{\prime}\left\{y_{1}\right\}=-\frac{6 \times 1 !}{s^{4}}, \quad L\left\{y_{1}\right\}=\frac{6 \times 1 !}{3 s^{3}}, \quad y_{1}=t^{2}, \\
& L^{\prime}\left\{y_{2}\right\}=-\frac{10 \times 3 !}{s^{6}}, \quad L\left\{y_{2}\right\}=\frac{10 \times 3 !}{5 s^{5}}, y_{2}=\frac{1}{2 !} t^{4}, \\
& L^{\prime}\left\{y_{3}\right\}=-\frac{7 \times 5 !}{s^{8}}, \quad L\left\{y_{3}\right\}=\frac{7 \times 5 !}{7 s^{7}}, \quad y_{3}=\frac{1}{3 !} t^{6}, \\
& L^{\prime}\left\{y_{4}\right\}=-\frac{3 \times 7 !}{s^{10}}, \quad L\left\{y_{3}\right\}=\frac{3 \times 7 !}{9 s^{9}}, \quad y_{3}=\frac{1}{4 !} t^{8},
\end{aligned}
$$

which is the expansion of the function $y(t)=\exp \left(t^{2}\right)$ and is the exact solution of example 6 .

The series solutions obtained by the MLDM are the same as Adomian decomposition method [28], variational iteration method [11] and homotopy perturbation method [9]. Unlike other approximation methods [14], [15], [27], [28], our method can get the exact solution. 


\section{CONCLUSION}

In this paper, we have successfully employed the Modified Laplace decomposition method (MLDM) to obtain exact solutions for singular IVPs of Lane-Emden-type. MLDM can accelerate the rapid convergence of series solution when compared with Laplace decomposition method. It is shown that the MLDM is a promising tool for singular IVP's of Lane-Emden type, and in some cases, yields exact solutions in two iterations.

\section{ACKNOWLEDGMENT}

This work is supported by National Natural Science Foundation of China (Grant Nos. 41105063).

\section{REFERENCES}

[1] S. Chandrasekhar, Introduction to the Study of Stellar Structure, New York: Dover, 1967.

[2] H. T. Davis, Introduction to Nonlinear Differential and Integral Equations, New York: Dover, 1962.

[3] O. U. Richardson, The Emission of Electricity from Hot Bodies, London: Zongmans Green and Company, 1921.

[4] A. M. Wazwaz, "A new algorithm for solving differential equations of Lane-Emden type,” Appl. Math. Comput., vol. 118, pp. 287-310, 2001.

[5] A. M. Wazwaz, "A new method for solving singular initial value problems in the second-order ordinary differential equations," Appl. Math. Comput., vol. 128, pp. 45-57, 2002.

[6] A. M. Wazwaz, "The modified decomposition method for analytic treatment of differential equations," Appl. Math. Comput., vol. 173, pp. 165-176, 2006

[7] M. S. H. Chowdhury and I. Hashim, "Solutions of a class of singular second-order IVPs by homotopy-perturbation method," Physics Letters A, vol. 365, pp. 439-447, 2007.

[8] M. S. H. Chowdhury and I. Hashim, "Solutions of time-dependent Emden-Fowler type equations by homotopy perturbation method," Physics Letters A, vol. 368, pp. 305-313, 2007.

[9] A. Yıldırım and T. Öziş, "Solutions of Singular IVPs of Lane-Emden type by Homotopy Perturbation Method," Physics Letters A, vol. 369, pp. 70-76, 2007.

[10] J. H. He, "Variational approach to the Lane-Emden equation," Appl. Math. Comput., vol. 143, pp. 539-541, 2003.

[11] A. Yıldırım and T. Öziş, "Solutions of singularIVPs of Lane-Emden type by the variational iteration method," Nonlinear Anal-Theor, 2009, vol. 70, pp. 2480-2484.

[12] A.-M. Wazwaz and R. Rach, "Comparison of the Adomian decomposition method and the variational iteration method for solving the Lane-Emden equations of the first and second kinds," Kybernetes, vol. 40, pp. 1305-1318, 2011

[13] V. S. Ertürk, "Differential transformation method for solving differential equations of Lane-Emden type," Math. Comput. Appl., vol. 12, pp. 135-139, 2007.

[14] R. K. Pandey, N. Kumar, A. Bhardwaj, and G. Dutta, "Solution of Lane-Emden type equations using Legendre operational matrix of differentiation," Appl. Math. Comput., vol. 218, pp. 7629-7637, 2012.

[15] A. Pirkhedri, P. Daneshjoo, H. H. S. Javadi, H. Navidi, S. Khodamoradi, and K. Ghaderi, "Generalized Laguerre Function Collocation Method Based on Transformed Hermite-Gauss Nodes," Int. J. Astron. Astrophys, vol. 1, pp. 67-72, 2011.

[16] S. A. Khuri, "A Laplace decomposition algorithm applied to a class of nonlinear differential equations," J. Appl. Math, vol. 1, no. 4, pp. $141-155,2001$.

[17] S. A. Khuri, "A new approach to Bratus problem," Appl. Math. Comput., vol. 147, pp. 31-136, 2004.
[18] M. I. Syam and A. Hamdan, "An efficient method for solving Bratu equations," Appl. Math. Comput., vol. 176, pp. 704-713, 2006.

[19] E. Yusufoğlu (Agadjanov), "Numerical solution of Duffing equation by the Laplace decomposition algorithm," Appl. Math. Comput., vol. 177, pp. 572-580, 2006.

[20] O. Kiymaz, "An algorithm for solving initial value problems using Laplace Adomian decomposition method," Appl. Math. Sci., vol. 3, pp. 453-1459, 2009.

[21] A. Wazwaz and M. S. Mehanna, "The Combined Laplace-Adomian Method for Handling Singular Integral Equation of Heat Transfer," I.J. of Nonlinear Science, vol. 10, pp. 248-252, 2010.

[22] F. A. Hendi, "Laplace Adomian Decomposition Method for Solving the Nonlinear Volterra Integral Equation with Weakly Kernels," Studies in Nonlinear Sciences, vol. 2, pp. 129-134, 2011.

[23] M. Khan and M. Hussain, "Application of. Laplace decomposition method on semi-infinite domain,” Numer. Algor., vol. 56, pp. 211-218, 2011.

[24] G. Adomian and R. Rach, "Noise terms in decomposition series solution," Comput. Math. Appl., vol. 24, pp. 61-64, 1992.

[25] M. Hussain and M. Khan, "Modified Laplace Decomposition Method," Applied Mathematical Sciences, vol. 4, pp. 1769-1783, 2010.

[26] S. A. Yousefi, "Legendre wavelets method for solving differential equations of Lane-Emden type," Appl. Math. Comput., vol. 181, pp. 1417-1422, 2006.

[27] J. I. Ramos, "Linearization techniques for singular initial-value problems of ordinary differential equations," Appl. Math. Comput., vol. 161, pp. 525-542, 2005.

[28] F. Olga and S. Zdenek, "Adomian decomposition method for certain singular initial value problems," Journal of Applied Mathematics, vol. III, pp. 91-98, 2010.

[29] M. Yiğider, K. Tabatabaei, and E. Çelick, "The Numerical Method for Solving Differential Equations of Lane-Emden Type by Padé Approximation," Discrete Dynamics in Nature and Society, pp. 1-9, 2011.

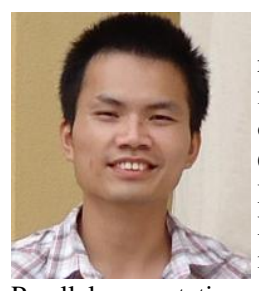

Fukang Yin was born in Guangxi, China, in 1984. He received the bachelor degree in applied mathematics from University of Electronic Science and Technology of China (UESTC) in 2008 and master degree in Computer Science from National University of Defense Technology (NUDT) in 2011. Now he is a $\mathrm{PhD}$ candidate student in NUDT. His main research interests are in numerical methods for PDEs and

Parallel computation.

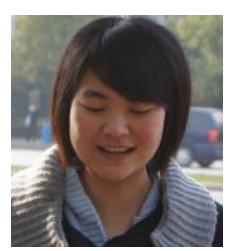

Wangyi Han was born in Shanxi, China, in 1990. She received the bachelor degree in Computer Science from University of Electronic Science and Technology of China (UESTC) in 2010. Now she is an enrolled postgraduate in National University of Defense Technology (NUDT). Her main research interests are in NWP and Parallel computation.

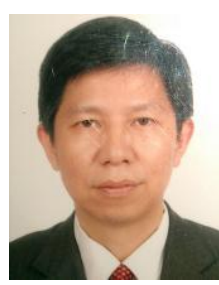

Junqiang Song was born in Hunan, China, in 1962 $\mathrm{He}$ received the bachelor and master degrees in Computer Science from National University of Defense Technology (NUDT) in 2011. Now he is a Professor in NUDT. His main research interests are in NWP and Parallel computation. 